Meta

Journal des traducteurs

Translators' Journal

\title{
Transfert de connaissances nouvelles et aménagement terminologique
}

\section{Monique Slodzian}

Volume 40, numéro 2, juin 1995

Usages sociaux des termes : théories et terrains

URI : https://id.erudit.org/iderudit/004170ar

DOI : https://doi.org/10.7202/004170ar

Aller au sommaire du numéro

Éditeur(s)

Les Presses de l'Université de Montréal

ISSN

0026-0452 (imprimé)

1492-1421 (numérique)

Découvrir la revue

Citer cet article

Slodzian, M. (1995). Transfert de connaissances nouvelles et aménagement terminologique. Meta, 40(2), 238-243. https://doi.org/10.7202/004170ar
Résumé de l'article

Les programmes d'aménagement linguistique destinés à pourvoir les langues qui ne participent pas au développement technologique en " vocabulaires modernes » ne tiennent pas suffisamment compte des contraintes propres à l'acquisition de concepts nouveaux. On se demandera si l'action terminologique menée sur ce terrain n'entretient pas l'illusion que l'appropriation des mots équivaut à l'appropriation des concepts. Or un simple retour en arrière dans les réalités populaires de notre avant-guerre montre que tous les actes importants de la vie s'articulaient sur des croyances qui ne se sont diluées que très lentement. Le nouveau s'est progressivement et incomplètement superposé à l'ancien. Aujourd'hui encore en France, un certain nombre de paysans continuent de semer à la nouvelle lune et de tailler les arbres à la lune descendante. Il faut revenir à l'aventure de la modernité dans notre propre culture lorsque l'on cherche à contribuer au transfert de connaissances nouvelles dans des cultures plus éloignées du monde technologique. Cela suppose la reconnaissance des langues et cultures autres. Parallèlement, I'étude de la formation des concepts abstraits chez I'adolescent met en lumière les contraintes cognitives auxquelles il faut satisfaire pour que l'apprentissage réussisse. On donnera quelques indications sur des pistes concrètes qui pourraient inspirer d'autres approches du transfert des connaissances.
Ce document est protégé par la loi sur le droit d'auteur. L'utilisation des services d’Érudit (y compris la reproduction) est assujettie à sa politique d'utilisation que vous pouvez consulter en ligne.

https://apropos.erudit.org/fr/usagers/politique-dutilisation/ 


\title{
TRANSFERT DE CONNAISSANCES NOUVELLES ET AMÉNAGEMENT TERMINOLOGIQUE
}

MoniQIJE: SLOOIZIAN

INALCO Pura Framie

\begin{abstract}
Résumé

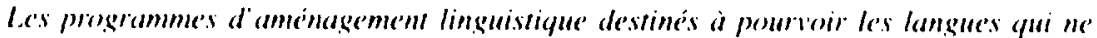

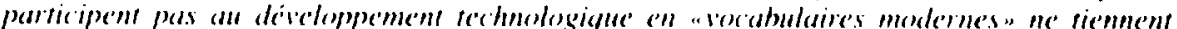

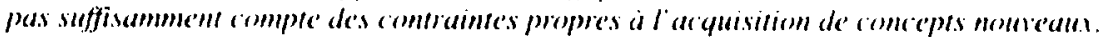

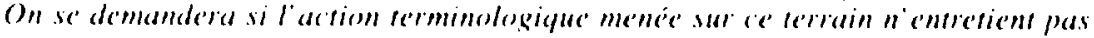

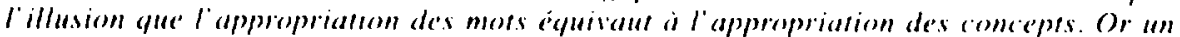

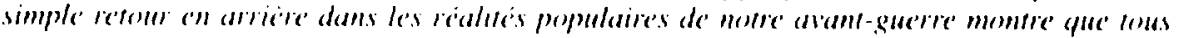

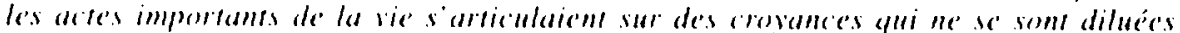

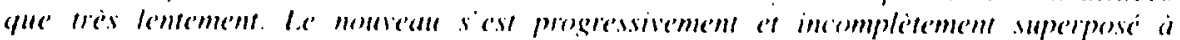

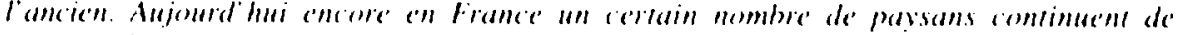

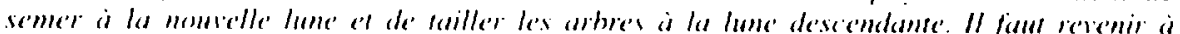

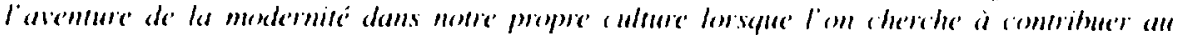

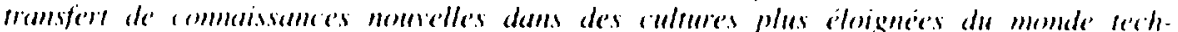

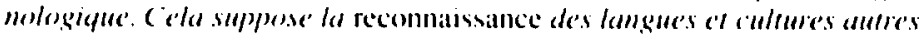

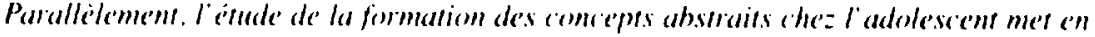

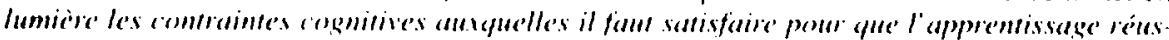

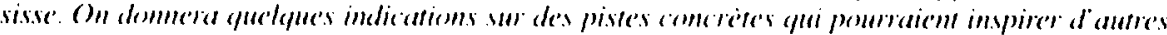
apporche's du ramsforl de's rommaissame's.
\end{abstract}

Qué gagne le pêcheur inuit à disposer du mot cance'r, obtenu par calque, emprunt ou autre mode de production néologique, s’il ne comprend pas le concept dénommé par le mot? Lal question n'est pas fortuite. A l'évidence, l'illusion que le processus d'acquisition des connaissances ressortit à des opérations de dénomination survit à ce que la philosophice et la psychologie de la connaissance ont établi depuis des décennies: la comprehension du nouveau n'est possible yu’a partir de lexpérience antérieure interprétée par notre système conceptuel indigène. Celui-ci est façonné par notre culture qui. en fin de compte. fabriguc le monde en en labriquant les catégories. Lat catégorisation est indispensable au raisonnement humain puisque nous raisonnons dans un monde possible, cohérent avec notre expérience, et non dans tous les mondes à la fois.

Cassirer voulait-il dire ature chose lorsqu il observait que:

ches lenfamt. le nom et ke savoir qui s'y rapportent jouent le même rôle que la main qui le guide dam la marche |... I. Arme du nom. il peut sessatyer à lat représentation des ohjets? (Cansirer 1933: 26)

Pour Piaget et Vygotski (Piaget 1926: Vygotski 1985). les peres de la psychologie cognitive, il était également acquis que l'apprentissage de savoirs nouveaux ne se fait jamais sur une table resse'.

L'illusion que l'on peut transmettre des connaissances nouvelles par des schémas verbaux vides pourrait bien être un avaltar de l'ethnocentrisme. qui procede justement de 
la difficulté à concevoir des mondes conceptuels autres. Par son immodestie. l'européocentrisme rend encore plus difficile la torsion nécessaire pour se rendre étranger à sa propre culture et à ses propres catégories de savoir. L'incompréhension est le résultat de ces atlituden figées. L'échec des campagnes d'aménagement linguistique en est la sanction.

Au-dela de ce constat banal se posent un certain nombre de questions cruciales pour le transfert de connaissances, notamment médicales, en direction de populations éloignées de la culture technologique occidentale.

\section{REPRESENTATIONS TRADITIONNELIES ET CONNAISSANC 'RS NOUVELILFS}

Dans la mesure où la production de connaissances scientifiques et techniques a pour foyer principal le monde occidental. les destinataires de ce savoir qui appartiennent à des cultures dites traditionnelles sont jugés par les émetteurs vierges de tout savoir "utile». Il s'agit bien du préjugé implicite sélon lequel les cultures en question ne catégorisent pas le réel à partir de l'observation, c'est-à-dire à partir de conduites cognitives qui pourraient bien être universelles. C'est méconnaitre que la chosce se'nsible constitue le point d'ancrage de la pensée indigcinc'. dimension de l'activité cognitive propre à chacun de nous, quelle que soit la culture. De l'expérience sensible sont issus les concepts spontanés à partir desquels se construit notre compréhension du monde. Si la construction des concepts scientifiques induit certainement une rupture avec l'expérience sensible, cela ne signifie pas que l'étape de la connaissance sensible soit facultative.

Chacun de nous sait que nos savoirs indigènes coexistent parallèlement aux connaissances proprement scientifiques. Si la majorité de la population des pays du monde occidental entretient un contact plus étroit avec l'univers technologique (médias, école. environnement, elc.), nul ne prétendra qu elle s'est «approprié» ces savoirs au point de les avoir substitués aux connaissances atraditionnelles». II suffit en effet de s"intéresser à la représentation des grands concepts de la physique. de la génétique ou de la biologie pour constater que la plupart dentre nous vivons avec des connaissances naives, largement périmées par less récentes avancées de ces disciplines.

Cette remarque en entraine une autre. La créolisation des connaissances à laquelle nous avons tous recours dans notre vie quotidienne participe largement du magique, au sens où nous utilisons des mots produits par la science sans en maîtriser la définition scientifique.

Ignace Meyerson notail que:

La tranquille et parfaite confiance en la permanence des lois de la nature n'est pas seulement le trait dominant de notre mentalité elle en est encore l'une des acquisitions les plus récentes. Loin d'être antéricure aux systèmes d'explication naturelle, elle en est le résultat: la sanction psychologique du success. (est parce que les sciences ont réussi que nous sommes sûrs qu elles réussiront. (Meyerson 1987:340)

Hors du monde rassurant des sciences, l'équilibre est en revanche fragile. On a réussi aujourd hui, mais demain? L'empirisme du succès explique le conservatisme des savoirs traditionnels. Ainsi, en intervenant dans notre univers quotidien, par le biais de la médecine notamment. les sciences dotent-elles l'homme occidental d'un système de croyance très efficace qui se substitue aux modèles explicatifs préscientifiques.

La puissance de la science occidentale tient en partie au fail qu'elle confère à ses populations plus de croyance que de savoir réel. Sa force identitaire est telle que les sujets en viennent à croire que leur conscience du monde $n^{\circ}$ a pas élé façonnée par des états antérieurs. Trouble de mémoire qui nourrit l'illusion de la «table rase» et n'est peut-être pas étranger à l'ignorance des cultures autres. 


\section{REALITE PHENOMENALE. FI MODE DF ( ATKGORISATION}

Dès lors que l’on admet que les paradigmes de la science contemporaine se sont construits progressivement à partir des connaissances protoscientifiques issues de l'observation et de l'expérimentation humaine, il semble raisonnable de présupposer un modèle général de cognition qui aclualise les mêmes types de conduite au-delà des différences culturelles (attention/observation empirique, codage de l'information. planification de l'action...). Les similarités que l'on constate entre les modes de classification populaire et scientifique des especes naturelles semblent indéniables, en dépit des réserves légitimes de certains anthropologues (Brown 1985: 4.3-64). L'hypothese d'un stalut cognitif commun aux taxinomies populaires et aux nomenclatures systématiques est actuellement discutée par de nombreux chercheurs (Hull 1992). Pour Atran (1990), de la Nouvelle-Cuinéce a New York, la façon dont les humains conceptualisent les especes suggère fortement l'existence d'un universel cognitit. Sans entrer dans le débat philosophique que la question appelle, et moyennant une certaine approximation dans la définition du concept. l hypothèse. telle qu'elle est formulec par Atran. nous interesse au premier chet.

Lintuition que nous fournit lélude de leminologies vernaculaires relatives à des savoirs médicaux, à la description de la nature et des êtres vivants en particulier, suggère bien la même conclusion. Ainsi la couche archaïque des concepts qui catégorisent à la fois le schéma corporel el les maladies provient-clle de l'expérience perceptuelle immédiate. La catégorisation de la maladie d après la partie du corps où elle se localise (mal de

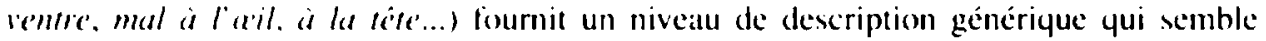
jouir d'une certaine universalité'. II en est de même de la catégorisation par symptômes

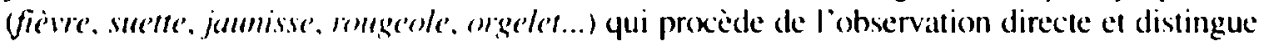
prioritairement l'aspect. la forme et la couleur. Les langues semblent donc encoder d'abord l'information dérivéc de l'observation de l'environnement.

La prédominance du perceptuel s'exprime dans le processus métaphorique. mode de compréhension dérivé du raisonnement analogique. Dans le travail cité plus haut, les données terminologiques décrivant l'anatomie, la physiologie de l'ail, ainsi que ses pathologies et symptômes dans lés quatre langues du Sahel ont été comparées aux résultats obtenus par Françoise Skodat dans son étude sur le vocabulaire de l'anatomie et de la pathologic en grec ancien (Skoda 198.3). Dans les cats grec el sahélien, on retrouve les catégories naturelles d'expérience liées à l'environnement qui servent de domaine source pour les dénominations et lés descriptions métaphoriques.

Cel universel cognitif qui procede du sems crmmmm, i.e. de propositions universelles partagées par tous sur le monde. sarrête au visible. à la réalité phénoménale du monde. Ainsi compris, le sens commun est une source de vérité pour la connatissance du monde expérienciel, mais il est faillible pour la connaissance de l'univers scientifique qui échappe à la perception. Ainsi la description populaire de l'ceil s'arrête-t-elle au nert optique. racinc de l'aril pour les langues sahéliennes.

Le primat de la réalité phénoménale peut aussi bien être constaté dans l'étymologie elle-même. Comme le précise Susan Sontag:

la définition la plus ancienne du cancer au sens propre le décrit comme une grosseur, une protubérance ou une excroissance et cette maladie selon Gallien, tirerait son nom - dérivé du grec karkinos signifiant "crabe" - de la ressemblance présentée par les veines gonflées d'une tumeur externe avec les pattes du crabe. Ee non, comme le croient beatucoup de gens. parce que les metaslatses gagnent sournoisement du terrain comme le ferait ceet animal. (Sontag 1977)

L'image basique de la protubérance se retrouve dans la tuberculose. Du latin ruberculum. diminutif de tuber. le mot désigne également une excroissance, une protubérance. Et 
jusqu’à une époque encore très récente, la tuberculose était - dans le domaine de la typologie - le cancer.

La confusion entre les deux maladies se retrouve chez les scientifiques eux-mêmes puisque Rudolf Virchow, qui a créé la pathologie cellulaire vers les années 1850, pensait que le tubercule était une tumeur (Sontag 1977).

Deux observations majeures trouvent ici leur place. Premièrement. le fondement perceptuel de la catégorisation des maladies explique que des pathologies perçues aujourd'hui comme presque contraires (tuberculose/cancer) n'ont été différenciées que tardivement. S'agissant du propos qui nous occupe - le repérage du champ d'interaction entre les deux cadres de pensées pour passer d'un système à l'autre - ces données devraient nous rendre plus attentifs à la logique des classifications vernaculaires. Ainsi, cataracte el glaucome sont-ils indifféremment désignés dans les langues du Sahel comme hrowillard ou v'oile. En haussa, on se réfère à l'araignée (gizo-gizo) pour exprimer la sensation de voile devant les yeux. Le chalazion et l'orgelet sont généralement désignés par des termes qui procèdent d'une logique analogue (boule, grain de mil, auf).

En second lieu, l'image basique imprimée dans les dénominations originelles (cancer) ne bloque pas le processus de reconceptualisation. L'image est susceptible d'évoluer et. tout au moins, de se neutraliser au profit d'un concept plus abstrait. Quels que soient les stéréotypes et les figures qui collent aux concepts "cancer" et "tuberculose». la tendance est. en gros, à l’objectivation, encore que la terreur de la peste démontre la prégnance des fantasmagories liées aux maladies exotiques...

La plasticité des désignations populaires suggère que l'on pourrait utiliser les termes des langues locales, si hous soient-ils, pour fixer les désignations en contormité avec la nosographie biomédicale. Il y a bien des chemins de traverse.

\section{L.F CHAINACIF DFS CONCEPTS}

Dans les premières années du $X X^{e}$ siècle, de célèbres médecins se sont intéressés de près aux résultats des campagnes d'hygiène dans la population française ravagée par le lléau de la tuberculose. La mise en lumière de l'existence des microbes (découverte du bacille par Koch en 1882) va modifier profondément la conception médicale de la contagion et la classification des maladies. Le changement de paradigme se fera très progressivement dans le corps médical. Quant à la population. elle adopte lentement la nouvelle terminologie sans changer de manière de voir:

|... Là où les gens avaient dit autrefois que l'ail chassait le mal. ils apprenaient à dire qu il chassait les microbes. Mais les microbes netaient que les esprits d'antan déguisés sous un nouveau nom. si hien que lorsque la maladie ravageait la cabane à lapins d'unc paysanne. elle meltait le cochon dedans, parce que sa mauvaise odeur tue ou éloigne les microbes. (Weber 198.3)

Les propagandistes de la lutte contre le bacille de Koch savaient qu'on ne gagnerait pas la guerre contre la tuberculose avec des mots. Ils reprenaient donc les représentations traditionnelles en définissant le microbe comme le mauvais génice. l'hôte mécommu de la maison... Ils avaient compris qu'il fallait infléchir le sens de traditions plus anciennes pour parvenir à la compréhension appropriée des phénomènes et. au-delà, pour induire de nouvelles conduites.

C'est ainsi qu'ils ont repéré l'importance du crachat dans les sociétés traditionnelles. Ils se sont appuyés sur celte tradition pour illustrer la notion de vectelur du bacille. A partir du crachat hacillifére, il est aisé d'expliquer les notions d'insalubrite (nids à poussière, ramages, alcôves, tapis, tentures et autres pièges à bacilles) et d'hygiène (le lisse et le clair). 
Le travail accompli dans le cadre de la lutte contre la tuberculose fut à maints égards remarquable. On notera en particulier que. confrontés à l'incompréhension massive des processus infectieux, les hygiénistes eurent à trouver une issue en s'appuyant sur la logique du raisonnement populaire. Le point de départ était un concept familier el concret présentant une forte charge symbolique. Ainsi, le concept de crachat était-il articulé sur un code définissant les comportements sociaux et les rapports à autrui.

Faut-il rappeler ici que l'enjeu du transfert de connaissances d'un système de pensée à un autre ne peut être la dénomination d'un seul concept isolé? La question est bien davantage de savoir comment un schéma-image initial peut devenir un vecteur correct d'information pour un fragment de connaissances. Ainsi l'OMS a-1-elle proposé de dénommer le glaucome "la maladie du rat». La métaphore du rat rongeant sournoisement le câble (i.e. le nerf optique) est en effet très efficace sur le plan cognitif puisqu elle pointe la caractéristique essentielle de la pathologie. à savoir l'évolution insidieuse du mal. Mais on se demandera si la rupture entre la dénomination par le symptôme (le voile. l'obscurité) vécu par le patient et la dénomination par l'étiologie (le processus de la pathologie) ont des chances égales. Le concept étiologique ne deviendra probablement opératoire qu après un très long travail.

Nous avons déjà évoqué le fait que dans les langues indo-européennes comme dans les langues sahéliennes étudiées, ce sont les symptômes, les signes externes en particulier. et la causalité supposée de la maladie qui sont à la source des dénominations. Faut-il chercher à introduire d autres logiques de dénomination? Quelles sont leurs chances de succès?

\section{AU-DELȦ DE L.A DF́NOMINATION}

L.ensemble de ces réflexions conduit à revoir le centre de gravité des processus de transfert des connaissances à travers les langues. Nous devons nous demander en particulier si le poids du nominalisme dans nos traditions linguistiques $n$ 'induit pas un souci excessif et réducteur de "l'imposition des noms». Le cadre de signification d'un concept dépasse le seul lexème de sa désignation. En lui subordonnant les autres éléments discursifs et visuels (explication, définition spontanée, métaphores associées...) qui concourent à la formation et à la compréhension d'un concept, on se trouve dans l'incapacité de dégager le modète de représentation sous-jacent.

Cette remarque en appelle une autre. Les recherches sur le vocabulaire de l'arithmétique en langue française à la fin du Moyen Âge (Benoit 1988), montrent que:

dans lensemble, malgre des exceptions, les substantifs dérivent direclement du latin alors que les verbes qui caractérisent les opérations relevent de la langue vulgaire. |... Tout se passe comme si la nécessite de donner une forme scientifique passait d'abord par le substantil qui est lidée, le concept. donc l'objet de la science et que le statut scientifique du vocabulaire nécessitait. pour la grande majorité. le recours à un langage dérivé directement du lattin. Le verbe, qui ne sert qu’à caractériser l'action peut resker, au moins un kemps, du ressort de la langue vulgaire.

La confrontation entre langue savante et langue populaire traduit un conflit qui se trouve au cour de notre débat: l'enjeu des transferts de vocabulaire ne doit pas être confondu avec celui du transfert des connaissances. Si la priorité est donnée au premier. il est impératif de considérer l'ensemble des moyens linguistiques et non linguistiques d'expression des concepts. "est à cette condition que l'on sera en mesure de produire des messages adéquats qui s'articulent sur les connaissances acquises.

\section{ELEMRNTS DR CONCLISSION}

Les modes de représentation que partage une société donnée se sont élaborés sur de très longues périodes historiques. Les résistances aux savoirs nouveaux sont sans doute 
constitutives des processus cognitifs humains. Les langues nous livrent les traces de la formation de nos connaissances. S'il est vrai que l'action néologique vise à populariser les connaissances nouvelles à travers les langues, la sémantique historique lui fournit une clé irremplaçable pour l'analyse des opérations cognitives.

Toutefois. l'analyse des seuls procédés de dénomination ne permet pas d’accéder aux modèles de représentation des concepts. Sans un travail de reconstitution du cadre discursif et sémiotique global, il est difficile d'accéder au chaînage des concepts qui structure un micro-domaine de savoir.

Cette approche de la fabrique linguistique des concepts présuppose un recentrage de l'objectif scientifique. le transfert des connaissances ne pouvant être confondu avec un simple transfert de vocabulaires.

\section{Notes}

1. Dans Slodsalan lá paraitre). On y trouvera l'analyse des terminologies rélatives à l'arl et a ses pathologues dams 4 langues du Sahel (bankara. Jarma. hausa. wolof).

\section{REFERENC WS}

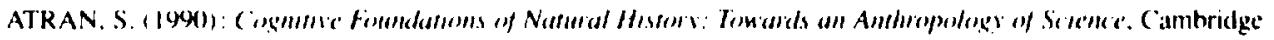
Iniversily Press

BI:NOIT. Paul (198X): "Langage populaire et langage savant : remarques sur la naissance du vocabulaire arith

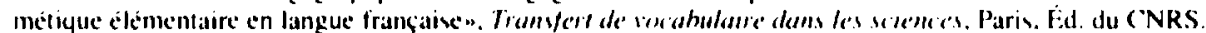

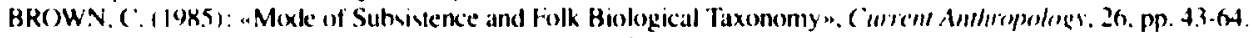

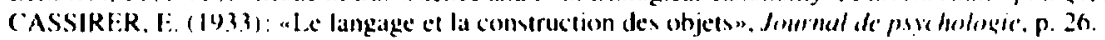

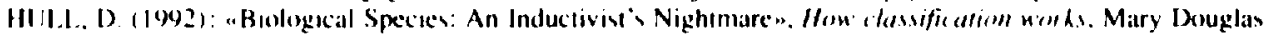
\& David I lull a Ed. ). Edinburgh. I:dinhurgh l Iniversity Prew

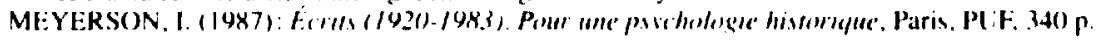

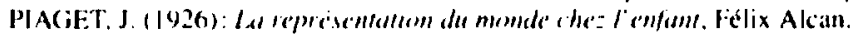

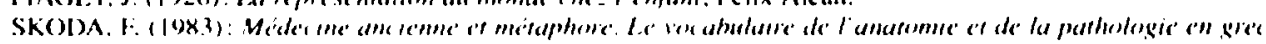
(m) u'). Temps actuel.

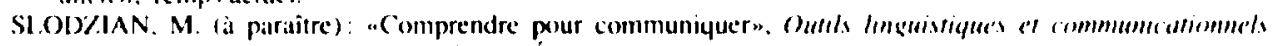
prow lo midharmm médirale. Paris, Didier Érudition.

SONTACi, S. (1977): La malade amme' misophowe, Irad. de I'anglais. Paris, Seuil.

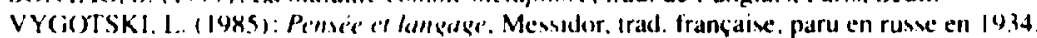

WEBER. E. (1963): La fin des lerrours. Parms. Fayard. 